\title{
¿Necesidad Económica o Preferencias Culturales? La Justificación Parental del Trabajo Infantil en Chile
}

\author{
Economic Constraints or Cultural Preferences? \\ The Parent Justification of Child Labor in Chile
}

\author{
María Francisca del Río y Patricio Cumsille \\ Pontificia Universidad Católica de Chile
}

\begin{abstract}
Con base en los datos de la Primera Encuesta Nacional sobre Trabajo Infantil realizada el año 2003 en Chile, se analizó la justificación parental para el trabajo de sus hijos. Se hipotetizó que se observaría una mayor probabilidad de justificación económica del trabajo infantil para los casos de mayor vulnerabilidad, es decir, niños y niñas de bajo nivel socioeconómico (NSE), menores de 12 años y el grupo de las niñas y adolescentes mujeres. El análisis de los datos, mediante una prueba de regresión logística, reveló que, si bien la necesidad económica efectivamente continúa siendo el principal motor del trabajo infantil en algunos grupos más vulnerables, las preferencias culturales y la participación de los adolescentes en esta decisión tienen un peso cada vez más importante.
\end{abstract}

Palabras clave: trabajo infantil, derechos del niño, axioma del lujo.

\begin{abstract}
Using data from the First National Survey on Children Labor conducted in 2003, we analyzed parental justification for children labor. We hypothesized that a higher probability of economic justification would be observed in parents of children from vulnerable groups: lower socioeconomic status (SES), children younger than 12 years, and girls. Logistic regression revealed that economic constraints are the most common justification among the more vulnerable groups, but that others reasons, such as cultural preferences and adolescence participation in the decision making process, are increasingly important.
\end{abstract}

Keywords: child labor, children's rights, luxury axiom.

Antecedentes

En las últimas dos décadas se ha observado a nivel mundial una mayor presencia de los derechos de los niños en el discurso público. Esto, sin duda, ha influido en un mejoramiento general de las condiciones de vida de los niños y niñas, pero algunos problemas aún persisten. Uno de estos es el trabajo infantil, que se concentra en las regiones más pobres del planeta (Basu \& Tzannatos, 2003).

\footnotetext{
María Francisca del Río, Programa Doctorado en Psicología, Pontificia Universidad Católica de Chile, Santiago, Chile. Patricio Cumsille, Escuela de Psicología, Pontificia Universidad Católica de Chile, Santiago, Chile.

La correspondencia relativa a este artículo debe ser dirigida a María Francisca del Río, Programa Doctorado, Escuela de Psicología, Facultad de Ciencias Sociales, Pontificia Universidad Católica de Chile, Vicuña Mackenna 4860, Macul, Santiago, Chile.E-mail: mndelrio@uc.cl
}

El trabajo infantil engloba un gran número de actividades que tienen diferentes consecuencias para los niños y niñas, labores que tienen distinto significado de acuerdo al contexto cultural en que se enmarcan. Esta amplitud de situaciones y contextos se traduce en la aplicación de múltiples definiciones de lo que se entiende por trabajo infantil, las que han producido importantes dificultades para medir y cuantificar el fenómeno y, por lo tanto, disímiles medidas de política pública destinadas a su erradicación.

Existe un importante consenso acerca de la pertinencia de diferenciar trabajo infantil del trabajo adolescente. La Organización Internacional del Trabajo (OIT) considera como trabajo infantil toda labor de tipo económico que es realizada por niños menores de 15 años, y como trabajo adolescente, el que es llevado a cabo por jóvenes entre 15 y 17 años ${ }^{1}$. Esta

1 A lo largo de este trabajo, como una manera de simplificar 
distinción es importante, pues el trabajo precoz suele tener efectos más negativos y permanentes, como la deserción del sistema escolar o mayores riesgos físicos y psicológicos (OIT, 2002). Concordante con esta diferenciación y sus implicancias, la legislación de la mayor parte de los países desarrollados prohíbe el trabajo de menores de 15 años ${ }^{2}$.

Datos de la OIT (2002) indican que alrededor de 250 millones de niños y adolescentes, entre 5 y 17 años, trabajan en el mundo, especialmente en los países en desarrollo. De esos, 20 millones de niños entre 5 y 14 años habitan en Latinoamérica y el Caribe. En Chile, en el año 2003 se llevó a cabo el primer estudio sobre trabajo infantil con una muestra representativa a nivel país, en el que se estimó que 196.104 niños y adolescentes chilenos $(5,4 \%$ de la población infantil) trabajaban ${ }^{3}$ y que $107.676(3 \%$ de la población infantil) lo hacían en condiciones inaceptables ${ }^{4}$ (OIT, Chile, Ministerio del Trabajo y Previsión Social [MINTRAB], Chile, Instituto Nacional de Estadísticas [INE] \& Servicio Nacional de Menores, 2004).

\section{¿Cuáles Son las Principales Consecuencias y Determinantes del Trabajo Infantil?}

La literatura existente sobre trabajo infantil da cuenta de distintos determinantes o causas asociadas a este problema, siendo la pobreza la que resulta

el discurso, nos referiremos a trabajo infantil englobando en esta expresión también el trabajo adolescente. Solo los distinguiremos cuando sea pertinente mencionar características diferenciales de uno u otro.

2 Chile ha adherido a esta política, prohibiendo el trabajo infantil.

3 La definición de niños ocupados se tomó de la encuesta de trabajo infantil utilizada en esta investigación. Esta encuesta consideró a los niños ocupados como aquellos que, teniendo entre 5 y 17 años, realizaban cualquier trabajo o actividad por el pago en dinero o en especies, o por la ganancia familiar, como trabajador no remunerado, y lo hacían por un período de tiempo de una hora o más, durante cualquier día de la semana de referencia.

4 El trabajo inaceptable, de acuerdo al Ministerio del Trabajo, incorpora una serie de condiciones: trabajar y ser menor de 12 años, niños entre 12 y 14 años que trabajen y no estudien, niños entre 12 y 14 años que trabajen más de 14 horas a la semana, trabajo adolescente por más de 21 horas a la semana no asistiendo a la escuela o por más de 49 horas semanales (lo que excedía para esa fecha el límite legal de jornada). También se incorporan todas las labores llevadas a cabo en la calle o en horario nocturno, sin importar la edad del niño o adolescente. universalmente más aceptada. Por otra parte, las consecuencias que el trabajo precoz tiene sobre los niños dan cuenta de la relevancia que tiene la búsqueda del entendimiento de las dinámicas asociadas a su causa y mantención.

Determinantes. Un estudio llevado a cabo por Ray (2000), utilizando datos de Pakistán, encontró que a mayor pobreza de los hogares se observaba mayor intensidad (mayor cantidad de horas) de trabajo infantil, como también una mayor deserción escolar asociada a la pobreza a través de la participación laboral de los niños. En otras palabras, de acuerdo a este estudio, la pobreza sería un determinante directo del trabajo infantil, y este lo sería de la deserción escolar.

Por su parte, Freije y Lopez-Calva (2000), utilizando datos de México y Venezuela para el período comprendido entre 1994 y 1998, observaron que la pobreza presentaba un efecto directo sobre el trabajo infantil en ambos países, por lo que concluyeron que la política más importante para erradicar el trabajo infantil era la lucha contra la pobreza.

Una de las hipótesis económicas más clásicas acerca de la relación entre trabajo infantil y pobreza es la introducida por Basu y Van (1998), con la idea del axioma del lujo (luxury axiom), que plantea que una familia enviará a sus niños al mercado de trabajo solo si los ingresos familiares provenientes de las fuentes laborales de los adultos caen a niveles que no permiten la subsistencia familiar. De este axioma se derivan dos ideas que retomaremos más tarde. La primera es que efectivamente la pobreza sería el principal determinante del trabajo infantil y la segunda, que serían los padres los que tomarían la decisión del ingreso de sus hijos al mercado laboral.

Pero la idea de la pobreza de la familias como principal factor que impulsaría el trabajo infantil es también una idea debatida y hasta polémica, enfrentándose los que defienden esta postura con los que plantean el trabajo infantil como un fenómeno más determinado por las preferencias y normas sociales (Edmonds, 2005). Favorecen a esta última hipótesis los casos de países desarrollados, donde también es posible encontrar trabajo infantil, aunque en menor escala, con otras características y asociado mayormente a necesidades de consumo de los niños y adolescentes (McKechnie \& Hobbs, 1999). En una investigación realizada con datos de Chile, Sapelli y Torche (2004) pusieron a prueba la hipótesis que la situación económica de la familia sería el determinante más importante del trabajo 
infantil. Los resultados mostraron que el ingreso no era una variable determinante en la decisión de trabajar, pero que sí lo era el género, pues los primeros en entrar al mercado laboral eran los niños y adolescentes hombres, los que efectivamente se encontraban trabajando en una mayor proporción que las mujeres. Estos hallazgos concuerdan con los de un estudio realizado en Perú, donde no se halló ninguna asociación entre la pobreza de los hogares y la cantidad de horas que los niños trabajaban (Ray, 2000).

De estos hallazgos se desprende que la idea de que la pobreza es considerada por algunos autores como la principal causa del trabajo infantil, es cuestionada por otros respecto a su poder predictivo, dando pie a hipótesis alternativas de tipo cultural para explicar el ingreso de niños y adolescentes al mercado laboral. Asimismo, se plantea que la decisión de que los niños trabajen tempranamente también se ve afectada por otros factores, tales como el sexo del niño.

Consecuencias del trabajo infantil. Respecto a las consecuencias que el trabajo infantil tiene sobre los niños, múltiples estudios han dado cuenta de cómo el trabajo precoz no permite a los niños beneficiarse de forma adecuada de la educación, tanto porque el trabajo les quita tiempo que no pueden dedicar a estudiar, como porque simplemente el trabajo induce a los niños a abandonar el sistema escolar formal (Emerson \& Portela, 2003; Fischman, 2003; Freije \& Lopez-Calva, 2000; Ray, 2000).

En efecto, en una extensa revisión de estudios acerca de las consecuencias del trabajo sobre los niños, Woodhead (2004) plantea que los niños que trabajan un importante número de horas se ven privados de experiencias y actividades consideradas clave para su desarrollo, principalmente de la escuela, lo que impacta negativamente sobre el desarrollo de habilidades cognitivas y sociales, lo que, a su vez, no les permite prepararse adecuadamente para la vida adulta. Asimismo, Woodhead observó que los niños se encuentran en mayor riesgo si el ambiente de trabajo no respeta su condición de seres en desarrollo, convirtiéndose así en un medio donde fácilmente se pueden presentar situaciones de abuso físico o sexual.

Por otra parte, un estudio de enfoque cualitativo llevado a cabo por la OIT y el Instituto Nacional de Estadísticas de Guatemala (INE) identificó importantes consecuencias que el trabajo infantil tiene sobre las vidas de los niños (Programa Internacional para la Erradicación del Trabajo Infantil [IPEC], OIT \& INE, 2003). Tomando como fuente el propio discurso infantil, se lograron observar consecuencias psicológicas, como depresión, ansiedad y pobre autoestima, efectos que se atribuían principalmente al hecho de asumir responsabilidades a temprana edad y a la realización de labores de alto riesgo.

\section{La Decisión Parental: Modelo de Restricciones Versus Preferencias}

Como se ha visto anteriormente, postular que la pobreza es el mayor determinante del inicio de los niños en el mercado laboral es una idea que provoca controversia entre los investigadores de esta área. Esto último da cuenta de la necesidad de estudiar otro tipo de mecanismos asociados al trabajo infantil, tales como los relacionados a la familia y cultura y su interacción con las fuerzas del mercado (Basu \& Tzannatos, 2003).

Dentro de una visión económica clásica, la participación de los niños en labores productivas se debe a la existencia de ciertas condiciones de mercado que permiten, ya sea por la decisión de las familias o la decisión de los propios niños, su incorporación a la fuerza de trabajo (MINTRAB, 2001).

Algunos estudios (Cerqueira \& Neder, 2001) muestran que los incentivos para el trabajo infantil provienen de las propias familias, y que otras veces la motivación nace de los propios intereses de los niños, niñas y adolescentes. En el primer caso, el trabajo infantil puede formar parte de una estrategia económica del grupo familiar que incentiva al niño a generar ingresos destinados a la economía doméstica; $y$ en el segundo, una generación de ingresos para sí mismo, destinados a financiar un tipo de consumo que no está al alcance de su familia (McKechnie \& Hobbs, 1999).

Pero, en todos los casos, una interesante característica de la participación de los niños y adolescentes en la vida laboral es que esta varía de acuerdo a su edad y sexo, el nivel de ingresos familiares, la educación de los padres y, para la mayoría de ellos, la decisión entre trabajar o estudiar es tomada por sus padres (Basu \& Van, 1998; Cerqueira \& Neder, 2001).

Por su parte, Amartya Sen (1999) plantea que la realización económica de las personas no se restringe a los bienes materiales que logren acumular sino, más bien, a la libertad que tengan para realizarse a través de ellos. Así, el juicio de una persona sobre 
su calidad de vida consiste en un juicio sobre su propia capacidad de conseguir logros que se consideran valiosos.

En relación a lo anterior, podemos suponer que las personas buscan una mejor calidad de vida $y$, por lo tanto, la mayor libertad para elegirla. En este marco se puede plantear que, en condiciones ideales, la decisión parental de que sus hijos trabajen refleja la búsqueda de logros que consideran valiosos, como podría ser la formación de destrezas, la adquisición de disciplina, la posibilidad de consumo, el hacer lo que todos hacen en un medio social $\mathrm{u}$ otras razones de tipo cultural o normativo. Así, cualesquiera sean las razones que impulsan a los padres a optar por el trabajo de sus hijos, ellas resultan prioritarias frente a la alternativa de poner en peligro el logro educacional de sus hijos que, como ya se planteó, entra en directa competencia con el trabajo infantil. Pero, por otra parte, en condiciones de urgente necesidad (económica) el que los niños trabajen no reflejaría sino una circunstancia que pasa por encima de las creencias y necesidades de la familia. Así, por ejemplo, esto explicaría que los niños trabajen, en desmedro de su formación educacional, a pesar de la valoración que los padres tengan de la educación.

De la discusión anterior se desprenden dos preguntas: los padres que no enfrentan severas necesidades económicas, ¿preferirían de todas formas enviar a sus hijos a trabajar? y ilos enviarían a trabajar, teniendo la oportunidad de elegir?

Se puede responder a estas interrogantes a través del modelo de preferencias versus restricciones de López- Calva (2001). Este modelo sigue la línea de lo planteado por Basu y Van (1998), pues toma como hipótesis que son las restricciones económicas las que imperan a la hora de promover el trabajo de los niños, ya que las preferencias de los padres rara vez explicarían el fenómeno. Esto último se entendería porque el trabajo infantil es una actividad que trae aparejada importantes consecuencias negativas para los niños (Emerson \& Portela, 2003). Así, aunque López-Calva postula que es la pobreza el mayor determinante del trabajo infantil y, por lo tanto, en la decisión de los padres tienen un mayor peso las restricciones, este modelo también da cabida a que el trabajo infantil se justifique por otro tipo de variables o preferencias, como pueden ser algunos factores culturales.

En Chile se aprecia la atingencia de un modelo teórico como el de López-Calva pues, si bien el trabajo infantil ocurre preferentemente en los estratos de menores recursos económicos (de los niños ocupados el $55,2 \%$ proviene de un NSE bajo, mientras que solo el $1,1 \%$ de un NSE alto), no todas las familias pobres permiten que sus hijos se incorporen al mercado laboral en forma permanente (de los niños ocupados de NSE bajo, solo el 46,4\% trabaja más de 30 horas semanales) (OIT et al., 2004). Asimismo, no todos los niños que trabajan provienen de familias que sufren importantes restricciones económicas. Esto da cuenta de factores culturales que incentivan el rol de las familias en la provisión de niños al mercado de trabajo (MINTRAB, 2001).

Entre los factores culturales que pueden ser parte de las preferencias parentales, podríamos hipotetizar que un rol destacado lo tienen razones vinculadas a necesidades de consumo por parte de los mismos niños, las que presionarían por lograr la aceptación familiar de su ingreso precoz al mercado laboral. Asimismo, la valoración de la educación a nivel familiar, vinculada estrechamente al nivel socioeconómico y educacional de los padres, es probable que marque una diferencia a la hora de permitir el trabajo de los niños de la familia, ya que una positiva valoración de la educación haría a los padres posponer la decisión de mandar a trabajar a sus hijos.

Otro factor cultural de importancia puede ser el sexo de los niños. Así, se podría esperar que el ingreso de las mujeres al mercado del trabajo se justifique mayormente por urgencias de índole económica. Así, por ejemplo, en el año 2003 en Chile, del total de niños ocupados, solo el 33\% eran mujeres, mientras que más del $90 \%$ de los menores de edad que realizaban quehaceres del hogar eran niñas (OIT et al., 2004). Por último, dado que la inserción laboral de los niños de menor edad es potencialmente más perjudicial para ellos, especialmente en términos de su educación, se podría esperar que mientras más pequeños sean los niños que participan del mercado laboral, la justificación parental esté más relacionada con motivos económicos. En relación a lo anterior, se ha establecido empíricamente que mientras menor es la edad de los niños al comenzar a trabajar, menos logros educacionales alcanzarán (López-Calva, 2001).

En este punto, también se deben tomar en cuenta otros factores asociados al funcionamiento de las familias como sistema, tales como la creciente participación que los niños tienen en la toma de decisiones familiares a medida que se acercan a la adolescencia. Desde esta perspectiva, la decisión de ingresar a la vida laboral tendría, entonces, una 
relación más directa con las necesidades de los adolescentes, si bien sería una decisión apoyada o aceptada por sus padres.

\section{¿La Decisión Es Parental? La Participación de los Niños y Adolescentes}

La literatura da cuenta que a medida que los niños crecen, y especialmente en la etapa de la adolescencia, ellos demandan un creciente nivel de autonomía en la toma de decisiones, lo que requiere que los padres ajusten su comportamiento, otorgándoles mayores niveles de autonomía. Así, los padres exitosos son capaces de entregar gradualmente mayores responsabilidades a sus hijos, tomando decisiones de forma menos unilateral (Criss, Laird, Pettit \& Bates, 2004).

En un estudio longitudinal, Criss et al. (2004) observaron que la trayectoria de las familias confirmaba una mayor participación de los adolescentes en la toma de decisiones familiares, pero que esta variaba en términos de los diferentes tipos de disciplina que las familias utilizaban, el rendimiento académico de los niños y el tipo étnico. Así, si bien para el general de los niños aumentaba su participación en la toma de decisiones familiares conforme se acercaban a la adolescencia, esta participación era relativamente menor en familias con padres de tipo más autoritario.

Por otra parte, datos recolectados en Estados Unidos muestran que la mayoría de los adolescentes declara participar junto a sus padres en la toma de decisiones familiares. Esta participación es mayor en las familias en las que los padres utilizan un estilo democrático de crianza. Así, estos padres suelen discutir con los niños asuntos que directamente afectan sus vidas, considerando su opinión en los procesos de decisión (Rodgers \& Small, 1997). Sin embargo, los adolescentes discriminan al momento de indicar las áreas en las que es legítimo que sus padres regulen su comportamiento (Smetana, 1988). De este modo, aun cuando los adolescentes tienden a reclamar mayor legitimidad sobre sus propias decisiones, ellos consideran legítimo que sus padres regulen aspectos de su vida que pueden afectar su desarrollo como, por ejemplo, fumar, usar drogas, relacionarse con personas problemáticas $\mathrm{y}$, en algunos casos, el tiempo que dedican a ver televisión (Cumsille, Darling, Flaherty \& Martínez, 2006). De esta forma, dado que el trabajo puede tener consecuencias negativas en su desarrollo, es probable que los adolescentes consideren legítimo que sus padres regulen esta área de su vida.

Así, en términos globales, la evidencia da cuenta de la participación creciente de los niños -acorde a su desarrollo- en la toma de decisiones que directamente les afectan, lo que cuestionaría la visión de una toma de decisión parental unilateral. Es probable, entonces, que la decisión de integrarse precozmente a la vida laboral sea una decisión compartida y que, por lo tanto, no se base solo en la percepción de las restricciones o preferencias por parte de los padres.

\section{¿Cómo Justifican los Padres el Trabajo de sus Hijos?}

A partir de lo expuesto, este trabajo se centrará en examinar la justificación que dan los padres respecto de la participación laboral de sus hijos menores de edad. Como ya se ha visto, esta justificación podría ser de índole económica, suponiendo que son las restricciones económicas las que operan con urgencia y frente a las cuales el trabajo de los niños es un precio necesario a pagar, a pesar de las consecuencias negativas que trae aparejado. Por otra parte, se ha visto que también es plausible una justificación más vinculada a las preferencias, o ideales de naturaleza más cultural; así, ideas como la necesidad de una formación laboral temprana o de las bondades de la autonomía económica de los niños y adolescentes podrían surgir como justificación.

Las consecuencias negativas que se asocian al trabajo infantil son un costo inevitable cuando la decisión se basa en argumentos de necesidad económica (restricciones); en cambio, para la justificación basada en preferencias, las consecuencias del trabajo infantil se convierten en un riesgo que vale la pena correr (el riesgo se evalúa como bajo). $\mathrm{Y}$, dado que los niños a medida que crecen hacen valer sus preferencias (y, consecuentemente, su poder de participar en las decisiones), también se debe tomar en cuenta que una justificación paterna basada en las preferencias incluye necesariamente la voz de los adolescentes.

Bajo este prisma, podríamos esperar que la justificación económica paterna o la basada en las preferencias presenten diferentes probabilidades de aparición cuando los niños que trabajan pertenecen a alguno de los grupos vulnerables. De esta manera, para los niños más pequeños, para quienes 
las consecuencias pueden ser más devastadoras, la justificación parental debiera estar relacionada estrechamente con razones económicas. En cambio, para el grupo de mayor edad la justificación paterna del trabajo infantil podría reflejar las preferencias, tanto propias como de sus padres. También se podría esperar una diferencia entre los distintos grupos socioeconómicos. Para los niños y adolescentes de familias de menores recursos económicos, se esperaría una mayor probabilidad de una justificación paterna que aluda a estas necesidades como la razón de ingreso de los niños al trabajo. Así, también, se puede esperar una diferencia de acuerdo al sexo de los niños, hipotetizando para la participación laboral de las niñas una justificación paterna más cercana a lo económico.

A partir de lo anterior, la principal hipótesis de este estudio es que la mayor probabilidad de justificación económica del trabajo infantil se observa entre los padres de niños ocupados de: NSE bajo, menores de 12 años y niñas y adolescentes mujeres. En contraposición, se espera entre los padres de NSE alto, de niños mayores de 12 años y mujeres, una menor probabilidad de justificación económica del trabajo de sus hijos. Esto último podría interpretarse como una decisión más cercana a las preferencias personales de los niños y adolescentes.

\section{Método}

\section{Datos}

Para la realización de esta investigación se contó con los datos de la encuesta sobre trabajo infantil recolectados en el primer trimestre del año 2003 en diferentes regiones de Chile por la OIT et al. (2004). Esta encuesta contó con una cobertura de 16.308 hogares y datos representativos de la realidad nacional.

Para el propósito de este estudio solo se utilizaron los datos provenientes del módulo que indaga acerca de las razones que llevaron a los niños a trabajar y las posibles consecuencias que esta actividad tiene para ellos (Módulo III D). La primera pregunta de este módulo consulta directamente al jefe de hogar acerca de las razones y justificación del trabajo de sus hijos, la segunda le pregunta sobre las consecuencias que tendría el hecho que sus hijos dejen de trabajar, y la tercera le consulta acerca de la actividad que preferiría que sus hijos lleven cabo: estudiar, trabajar o ambas ${ }^{5}$.

Se utilizaron solo los datos de la población de niños declarados como ocupados, o sea, económicamente activos, pues es esta actividad la que plenamente concuerda con la definición de la OIT respecto del trabajo infantil y son los datos que nos permiten responder la pregunta de esta investigación. Esta elección dejó fuera los datos de los niños y niñas (en su mayoría mujeres) que realizan quehaceres del hogar, labor que pareciera funcionar bajo otros supuestos y que puede ser parte del análisis de futuras investigaciones. Los datos permiten llevar a cabo un análisis diferenciado por sexo, NSE y edad.

Esta encuesta determinó el NSE en base a distintas variables relacionadas con la vivienda y la familia: el nivel de hacinamiento, el ingreso medio del hogar, la educación y el trabajo del jefe de hogar. A partir de esto se clasificaron los hogares en cinco grupos de mayor a menor pobreza: bajo, medio-bajo, medio, medio-alto y alto. Con el fin de simplificar el análisis descriptivo de los datos, en la presente investigación se recodificó el NSE, combinando en una categoría el nivel bajo y el medio-bajo, en otra categoría se mantuvo el nivel medio y en una tercera categoría se fusionaron los niveles medio-alto y alto.

Las tres preguntas utilizadas de la encuesta sobre trabajo infantil presentan distintas alternativas de respuesta. El jefe de hogar sólo debía responder una de ellas en cada pregunta. Para responder acerca de la existencia de dos clases de decisión o justificación del trabajo infantil, fue necesario agrupar las alternativas de respuesta de acuerdo a qué tipo de decisión reflejaban. Por ejemplo, frente a la pregunta ¿cuál es la razón principal por la que usted permite que su hijo/a trabaje?, las únicas alternativas que inequívocamente reflejan una decisión basada en necesidades económicas del grupo familiar son "para que ayude a pagar los gastos..." y "porque debe ayudar en nuestro trabajo". Una segunda categoría de respuestas que podría también asociarse a necesidad económica es: "porque iba a tener un hijo", "para que tenga un oficio desde joven", "porque quería trabajar" y "porque dejó los estudios". Sin embargo, estas alternativas de respuesta ciertamente no dejan traslucir una urgencia familiar de subsistencia y dejan espacio para interpretarlas como preferencias

\footnotetext{
La encuesta completa, que contiene el módulo III D, se puede encontrar en www.trabajoinfantil.cl
} 
paternas o del propio niño/a. Este grupo de respuestas puede ser parte de una categoría que podríamos llamar intermedia ya que, por ejemplo, que la razón del trabajo de los niños sea que ellos mismos quieren trabajar, parece una decisión guiada por sus propias preferencias pero, también, puede reflejar que frente a la necesidad económica familiar es más fácil para los padres desplazar la decisión del trabajo infantil a sus hijos que asumirla como propia. Asimismo, las alternativas "para que tenga un oficio desde joven" y "porque dejó los estudios" podrían interpretarse como una declaración de principios de los padres, pero en el fondo reflejar una racionalización de la necesidad económica que lleva a la inserción laboral de los hijos.

Por último, una tercera categoría sí refleja claramente que son las preferencias culturales de los padres las que guían la decisión que los niños trabajen, a saber, "para que se aleje de las malas compañías" y "para que sepa lo que cuesta ganarse la vida" (ver las categorías en el Anexo).

En las preguntas 2 y 3 las alternativas "no tendríamos dinero suficiente para vivir", "el negocio del hogar no podría funcionar", que describen las consecuencias de la posibilidad que los hijos dejen de trabajar, denotan una justificación económica, como también lo hace la preferencia de que los niños/as "sólo estudien" (ver el Anexo).

\section{Plan de Análisis}

Este estudio está dirigido a identificar las variables que de mejor forma predicen la justificación que los padres hacen del trabajo de sus hijos, justificación que puede ser, de acuerdo a la teoría, basada en criterios económicos o de preferencias. Debido al uso de una variable dependiente dicotómica -justificación económica versus preferencias- se realizó un análisis estadístico de regresión logística para responder a las preguntas de esta investigación (Pedhazur, 1997). Se llevó a cabo una regresión logística binaria, utilizando el método "enter" para determinar cuáles de las variables predictoras, NSE, sexo del niño/a y edad, eran las que mejor explicaban el tipo de justificación parental, como también qué tan preciso es el modelo propuesto para clasificar la justificación parental en los tipos económica o de preferencias. Este análisis se llevó a cabo con el paquete estadístico SPSS 11.5.
Resultados

\section{Análisis Descriptivo}

Los porcentajes de respuesta a las diferentes alternativas de las preguntas del Módulo III D por parte de todos los padres de niños ocupados se pueden observar en la Tabla 1.

En la mayoría de las respuestas aparece una tendencia claramente observable: los padres no responden masivamente a las alternativas que caracterizan una decisión de índole económica o de preferencias. En la pregunta 1 (razones y justificación del trabajo de su hijo/a) los porcentajes más altos de respuesta se observan en las alternativas "para que tenga su propio dinero" (24\%) y "porque quería trabajar" $(18 \%)$. Por otra parte, las alternativas que se categorizaron como una decisión económica solo suman entre ellas un $20 \%$ de las respuestas a la pregunta 1 , mientras que las que representan claramente una decisión de preferencias suman un 13\%.

En la pregunta 2, solo el 9\% respondió las alternativas relativas a una decisión económica, mientras que el $75 \%$ de los padres eligió alternativas que reflejan una decisión basada en preferencias: "no pasaría nada si es que los niños dejan de trabajar". Por último, solo para el caso de la pregunta 3 , en la que se consulta acerca de lo que preferirían que hicieran sus hijos, el $78 \%$ de los padres se inclina por la opción que refleja una decisión, de acuerdo a la teoría, de naturaleza económica: "que solo estudien".

Los porcentajes antes comentados nos muestran una tendencia que refleja que, a la hora de decidir que los hijos comiencen a trabajar, no son las urgencias económicas las que priman sino que es posible que tengan un mayor peso razones ligadas a la autonomía del niño-adolescente o a preferencias de los padres. Esto se refleja especialmente al preguntar qué sucedería si el niño/a deja de trabajar, a lo que el 75\% respondió que "no sucedería nada". Pero, por otra parte, de acuerdo a las frecuencias de respuesta a la pregunta 3, el 78\% de los padres desearía que sus hijos solo estudiaran, lo que resulta contradictorio pues, si bien el trabajo de sus hijos parece no ser indispensable para la supervivencia de la familia y quisieran que se dedicaran solo a estudiar, la realidad nos muestra que los niños están trabajando a pesar que esta actividad compite directamente con un buen desempeño escolar. Entonces, surgen las preguntas, 
Tabla 1

Porcentajes de Respuestas a Alternativas de Preguntas del Módulo III D

\begin{tabular}{|c|c|}
\hline $\begin{array}{l}\text { Alternativas de Respuesta a la Pregunta 1: Razones } \\
\text { para el trabajo infantil }\end{array}$ & Porcentaje de Respuesta \\
\hline Para que ayude a pagar los gastos & 12 \\
\hline Para que se aleje de malas compañías & 6 \\
\hline Para que tenga su propio dinero & 24 \\
\hline Para que tenga un oficio desde joven & 8 \\
\hline Porque quería trabajar & 18 \\
\hline Porque iba a tener un hijo & 0 \\
\hline Porque dejó los estudios & 2 \\
\hline Para que sepa lo que cuesta ganarse la vida & 7 \\
\hline Porque debe ayudar en nuestro trabajo & 8 \\
\hline Otra & 15 \\
\hline Total & 100 \\
\hline \multicolumn{2}{|l|}{$\begin{array}{l}\text { Alternativas de Respuesta a la Pregunta 2: Qué } \\
\text { pasaría si el hijo/a deja de trabajar }\end{array}$} \\
\hline No tendríamos dinero suficiente para vivir & 8 \\
\hline El negocio del hogar no podría funcionar & 1 \\
\hline No aprendería a trabajar & 9 \\
\hline No pasaría nada & 75 \\
\hline Otra & 7 \\
\hline Total & 100 \\
\hline \multicolumn{2}{|l|}{$\begin{array}{l}\text { Alternativas de Respuesta a la Pregunta 3: Cuál } \\
\text { sería su preferencia para su hijo }\end{array}$} \\
\hline Solo estudiar & 78 \\
\hline Solo trabajar & 1 \\
\hline Estudiar y trabajar & 15 \\
\hline Estudiar y realizar los quehaceres del hogar & 2 \\
\hline Hacer los quehaceres del hogar & 0 \\
\hline Poner un negocio propio & 0 \\
\hline Buscar un mejor trabajo & 1 \\
\hline Otra & 3 \\
\hline Total & 100 \\
\hline
\end{tabular}

¿son los mismos niños y adolescentes quienes finalmente deciden trabajar, a pesar del deseo de sus padres? ¿este fenómeno cambia de acuerdo al NSE de origen, la edad o sexo de los niños?

Al contrastar las tendencias de respuestas observadas para el total de los niños ocupados con las que aparecen en cada uno de los factores sobre los que se hipotetizó (edad, sexo y NSE), se pueden observar algunos patrones interesantes.

La proporción de respuesta a la pregunta 1 que afirma que "los niños deben ayudar en nuestro trabajo" es diferenciada por sexo. Mientras solo el 6\% de los padres escoge esta alternativa cuando se trata de hijos hombres, el 13\% lo hace cuando se trata de hijas mujeres. Si bien ninguno de los porcentajes es alto, es el doble para las mujeres que para los hombres. De esto se puede suponer una tendencia a elegir en primer lugar a las mujeres para ayudar en un negocio familiar.

Si revisamos las respuestas para los tres tramos de NSE en que se consignaron los datos -bajo, medio y alto-, la opción que los niños trabajen para ayudar a pagar los gastos aparece solo en el NSE bajo (con un $15 \%$ ), sin presentarse en el NSE medio o alto, lo que confirma la presunción que a menores ingresos está más presente la justificación del trabajo infantil por necesidades económicas. Esta idea se confirma al observar que la consecuencia familiar de que "no 
tendríamos dinero suficiente para vivir" si el niño dejase de trabajar, se observa también solo en el NSE bajo (13\%). Por otra parte, la opción "para que sepa como ganarse la vida" solo aparece en el NSE alto, con un $22 \%$, lo cual reflejaría la condición más acomodada y de privilegio en que se desarrollan estos jóvenes. En cambio, que no aparezca este tipo de justificación en los NSE medio y bajo probablemente refleja que las condiciones de vida más duras hacen evidente las dificultades de subsistencia.

Por último, al revisar los datos según la edad que los niños tenían al comenzar a trabajar, podemos ver que para los que comenzaron más temprano -antes de los 12 años-, las alternativas que reflejan una decisión económica no presentan una proporción de respuesta significativamente diferente a la de otros grupos etarios.

\section{Regresión Logística}

Para llevar a cabo este análisis estadístico, en primer lugar, se dicotomizó la variable dependiente $\mathrm{y}$ algunas de las variables independientes. La variable dependiente, tipo de justificación del trabajo infantil, se construyó en base a las alternativas de respuesta a la pregunta 1 (en la que se consulta al padre acerca de la razón por la que permite el trabajo de su hijo/a) que claramente daban cuenta de una decisión económica o de preferencias. Tal como se justificó con anterioridad, se clasificaron como razones que reflejaban una decisión económica las que daban realmente cuenta de una urgencia económica insalvable, y como razones de preferencia, las que indiscutiblemente mostraban este espíritu (ver la categorización de alternativas en el Anexo). En cuanto a las variables independientes, el NSE de los niños y niñas se dicotomizó en bajo y medio, pues el número de casos en el NSE alto no era significativo para este tipo de análisis. El sexo y la edad se dicotomizaron en hombre-mujer y menor de 12 años-12 años y más, respectivamente ${ }^{6}$.

Tomando en cuenta solo las categorías que reflejaban claramente una justificación económica o de preferencias, el análisis de regresión logística incluyó 64.934 niños y niñas ocupados.

Los resultados de la regresión logística se pueden observar en la Tabla 2.

\section{Tabla 2}

Regresión Logística para Predecir Decisión Económica de los Padres para Permitir el Trabajo Infantil de los Hijos, según NSE, Edad y Sexo

\begin{tabular}{lcccc}
\hline Predictores & $B$ & E.S. & Sig. & ODD \\
\hline NSE bajo & 0,261 & 0,013 & 0,000 & 1,793 \\
Edad 12 años y más & 1,247 & 0,022 & 0,000 & 3,480 \\
Sexo mujer & 0,883 & 0,020 & 0,000 & 2,480 \\
\hline
\end{tabular}

E.S.: error standard

Como se puede apreciar en la Tabla 2, todas las variables independientes del modelo son significativas para predecir el tipo de justificación parental del trabajo infantil $(p=0,000)$. El ODD de que los padres de NSE bajo utilicen una justificación económica es 1,79 mayor que el ODD de los padres de NSE medio (IC': $1,74-1,83$ ). En cuanto a la edad de los hijos, el ODD de usar una justificación económica de los padres de niños que comenzaron

\footnotetext{
6 Se decidió este corte etario pues es el que propone el Gobierno de Chile para distinguir el trabajo inaceptable del que no lo es.

7 Intervalo de confianza del ODD.
}

a trabajar después de los 12 años es 3,4 mayor que el ODD de los padres de niños que comenzaron su vida laboral antes de los 12 años (IC: 3,33 - 3,63).

Especialmente interesante es el caso de las niñas que trabajan, pues el ODD de que sus padres utilicen una justificación económica es 2,4 veces mayor que el de los padres de hijos varones que trabajan (ODD = 2,4; IC: 2,32 - 2,51).

Los resultados de la regresión mostraron que el modelo de tres variables predictoras (NSE, sexo y edad) explica el $8 \%\left(R^{2}\right.$ de Cox y Snell: 0,087$)$ de la variabilidad de la justificación parental del trabajo infantil. Si bien el modelo fue capaz de clasificar correctamente el $65 \%$ del total de casos, clasificó 
correctamente el 90\% de los casos de decisión económica y solo el $25 \%$ de los casos con justificación de preferencias. Esto demuestra que el modelo clasifica correctamente los casos en los que la decisión es económica, pero lo hace en forma deficitaria en los casos que reflejarían una decisión basada en preferencias, lo que se convierte en una limitación para la interpretación de los datos. Lo anterior podría deberse al amplio abanico de alternativas que se englobaron en la definición de la categoría no económica (o preferencias). En otras palabras, es posible que las justificaciones del trabajo infantil que se escogieron para integrar la variable de decisión basada en preferencias no tuvieran entre ellas una sustantiva relación de base.

\section{Discusión Final}

Los porcentajes de respuesta observados para las razones dadas por los padres para el trabajo de sus hijos no parecen clarificar una tendencia hacia una justificación económica o de preferencias. La mayor parte de las respuestas se concentra en alternativas que en una primera impresión pueden reflejar la idea del trabajo infantil como una situación electiva, en la que el niño o adolescente es quien decide trabajar para tener sus propios recursos. Sin embargo, no se puede afirmar que estas alternativas no escondan la necesidad de conseguir recursos a través del trabajo de los niños, con el fin de cubrir algunas necesidades básicas que los padres no alcanzan a solventar con su propio trabajo.

La tendencia de respuesta a las dos preguntas restantes parece confirmar que no es precisamente la urgencia económica la que guía la decisión familiar de que los niños y niñas trabajen. Si bien las alternativas de respuesta más populares no permiten confirmar esta conjetura, pues no son lo suficientemente directas en el sentido de reflejar un tipo de decisión u otra, la tendencia de respuesta observada en relación a la pregunta de lo que pasaría si el hijo deja de trabajar sí lo hace, en la dirección de una justificación del trabajo infantil basada en preferencias.

De lo anterior podríamos concluir la existencia de un patrón de la justificación del trabajo infantil en base a normas de crianza o usos culturales. Tienen especial fuerza las propias necesidades y opciones de los niños/adolescentes: querer trabajar o tener dinero propio.

Por otra parte, llama la atención la altísima proporción de padres a quienes les gustaría que sus hijos solo estudiaran, lo que resulta una suerte de contradicción con el hecho que permitan que sus hijos trabajen, especialmente si no lo hacen por estricta necesidad. Esto se podría interpretar bien como una respuesta de deseabilidad social frente al encuestador, pues en nuestro país está instalado el discurso de la importancia de la educación en la formación de las personas (MINTRAB, 2001), o bien como falta de consistencia con sus creencias. Por otra parte, podría también entenderse como la imposición de la voz de los niños/adolescentes en este tipo de decisiones: ellos son los que quieren trabajar.

En todo caso, estos datos parecen no apoyar las teorías que ubican a la necesidad económica familiar como el principal motor del trabajo infantil sino que apoyan las teorías alternativas de quienes plantean que el fenómeno del trabajo infantil se apoya en otras dinámicas más ligadas a los fenómenos de consumo y autonomía adolescente.

Los datos que distinguen ciertos patrones para los diferentes sexos, el NSE y horas trabajadas a la semana, sí tienden a confirmar que la justificación económica se presenta más marcadamente en algunos grupos vulnerables: los más pobres y las mujeres. Este último fenómeno se ve confirmado por los datos resultantes de la regresión logística, los que refuerzan los postulados teóricos de la teoría del axioma del lujo pues, efectivamente, son algunos de los grupos más vulnerables de la sociedad, las familias pobres y las niñas, quienes presentan una mayor probabilidad de tomar la decisión de integrarse al mercado laboral, basada en criterios económicos. Así, son las familias más pobres, y más especialmente con hijas mujeres, las que atrasan la decisión de que sus hijos trabajen hasta el punto que solo sea necesario y/o urgente hacerlo para la supervivencia económica del núcleo familiar (López-Calva, 2001).

Los datos de la encuesta utilizada no permitieron llevar a cabo otros análisis estadísticos ni otorgar una mayor potencia a los realizados, por lo que se aconseja realizar futuros estudios que resulten más conclusivos en relación a este fenómeno. Dentro de una línea de investigación futura, en las próximas encuestas podría formularse un mayor número de preguntas especialmente dirigidas a comprender este 
fenómeno, consultas que, basadas en la evidencia que la presente investigación aporta, permitan responder si la justificación del trabajo infantil efectivamente es diferente entre los grupos vulnerables y los que no lo son y, asimismo, aporten mejores indicadores de una decisión basada en preferencias.

Esta investigación sí fue capaz de identificar un patrón interesante respecto a las razones del trabajo infantil: existe evidencia que las razones económicas aún son el principal motor en algunos grupos más vulnerables, pero las preferencias culturales y la participación de los adolescentes en esta decisión van ganando terreno $\mathrm{y}$, ciertamente, ya no se puede afirmar que el trabajo infantil solo se justifica, en el ámbito familiar, por la urgencia de la supervivencia económica. Por lo tanto, una interesante veta de estudio futuro es profundizar en las razones culturales-preferencias que se encuentran detrás de la decisión familiar del trabajo infantil y, más especialmente, en la participación creciente de los adolescentes y las razones que los guían para ingresar al mercado laboral.

\section{Referencias}

Basu, K. \& Tzannatos, Z. (2003). Child labor and development: An introduction. The World Bank Economic Review, 17, 145-146.

Basu, K. \& Van, P. H. (1998). The economics of child labor. The American Economic Review, 88, 412-427.

Cerqueira, G. \& Neder, G. (2001). Social and historical approaches regarding street children in Rio de Janeiro (Brazil) in the context of the transition to democracy. Childhood, 8 , 11-29.

Criss, M. M., Laird, R. D., Pettit, G. S. \& Bates, J. E. (2004, Marzo). Autocratic parenting in adolescence: An examination of antecedents and developmental trajectories. En J. L. Kerpelman (Coord.), Person-centered analysis strategies: A diverse approach to adolescent research. Simposio realizado en la Biennial Meeting of the Society for Research in Adolescence, Baltimore, Estados Unidos. Extraído el 26 Junio, 2006, de http://www.cdp.auburn.edu/conference\%20presentations/ Biennial $\% 20$ Meeting $\% 20$ of $\% 20$ the $\% 20$ Society $\% 20$ for $\% 20$ Research\%20in\%20Adolescence\%20Baltimore,\%20MD,\%20 March\%202004/CrissSRA2004.pdf
Cumsille, P., Darling, N., Flaherty, B. P. \& Martínez, M. L. (2006). Chilean adolescents' beliefs about the legitimacy of parental authority: Individual and age-related differences. International Journal of Behavioral Development, 30, 97-106.

Chile, Ministerio del Trabajo y Previsión Social. (2001). Plan de Prevención y Erradicación Progresiva del Trabajo Infantil y Adolescente en Chile. Santiago, Chile: Autor.

Edmonds, E. V. (2005). Does child labor decline with improving economic status? The Journal of Human Resources, 40, 77-99.

Emerson, P. M. \& Portela, A. (2003). Is there a child labor trap? Intergenerational persistence of child labor in Brazil. Economic Development and Cultural Change, 51, 375-398.

Fischman, G. E. (2003). Children's work, schooling and welfare in Latin America. Contemporary Sociology, 31, 110-125.

Freije, L. \& López-Calva, L. F. (2000). Child labor, school attendance and poverty in Mexico and Venezuela (Documento de Trabajo No 1-2001). México, D. F.: El Colegio de México.

López-Calva, L. F. (2001). Child labor: Myths, theories and facts. Journal of International Affairs, 55, 59-73.

McKechnie, J. \& Hobbs, S. (1999). Child labour: The view from the North. Childhood, 6, 89-100.

Organización Internacional del Trabajo (2002). Panorama regional del trabajo infantil. Lima: Autor.

Organización Internacional del Trabajo, Chile, Ministerio del Trabajo y Previsión Social, Chile, Instituto Nacional de Estadísticas \& Servicio Nacional de Menores (2004). Trabajo infantil y adolescente en cifras: sintesis de la primera encuesta nacional y registro de las peores formas. Santiago, Chile: Organización Internacional del Trabajo.

Pedhazur, E. (1997). Multiple regression in behavioral research ( $3^{\mathrm{a}}$ ed.). New York: Holt, Rinehart \& Winston.

Programa Internacional para la Erradicación del Trabajo Infantil, Organización Internacional del Trabajo \& Guatemala, Instituto Nacional de Estadísticas (2003). Informe para Guatemala. Trabajo infantil: la percepción de los niños. Ciudad de Guatemala: Organización Internacional del Trabajo.

Ray, R. (2000). Child labor, child schooling, and their interaction with adult labor: Empirical evidence for Peru and Pakistan. The World Bank Economic Review, 14, 347-367.

Rodgers, K. B. \& Small, S. A. (1997). Living with your teenager: The changing parent-child relationship (NCR Publications $\mathrm{N}^{\mathrm{o}} 121$ ). Madison, WI: University of Wisconsin-Extension Cooperative Extension.

Sapelli, C. \& Torche, A. (2004). Deserción escolar y trabajo juvenil: ¿dos caras de una misma decisión? Cuadernos de Economía, 41, 173-198.

Sen, A. (1999). Nuevo examen de la desigualdad. Madrid: Alianza.

Smetana, J. (1988). Adolescents' and parents' conceptions of parental authority. Child Development, 59, 321-335.

Woodhead, M. (2004). Psychosocial impacts of child work: A framework for research, monitoring and intervention. The International Journal of Children's Rights, 12, 321-378. 
Anexo

Clasificación de las Respuestas del Módulo III D

\begin{tabular}{lc}
\hline Alternativas de Respuesta Pregunta 1 & Tipo de Decisión \\
\hline Para que ayude a pagar los gastos & Económica \\
Para que se aleje de malas compañías & Preferencias \\
Para que tenga su propio dinero & Intermedia \\
Para que tenga un oficio desde joven & Intermedia \\
Porque quería trabajar & Intermedia \\
Porque iba a tener un hijo & Intermedia \\
Porque dejó los estudios & Intermedia \\
Para que sepa lo que cuesta ganarse la vida & Preferencias \\
Porque debe ayudar en nuestro trabajo & Económica \\
\hline Alternativas de Respuesta Pregunta 2 & \\
\hline No tendríamos dinero suficiente para vivir & Económica \\
El negocio del hogar no podría funcionar & Económica \\
No aprendería a trabajar & Intermedia \\
No pasaría nada & Preferencias \\
\hline Alternativas de Respuesta Pregunta 3 & \\
\hline Solo estudiar & Económica \\
Solo trabajar & Preferencias \\
Estudiar y trabajar & Intermedia \\
Estudiar y realizar los quehaceres del hogar & Preferencias \\
Hacer los quehaceres del hogar & Preferencias \\
Poner un negocio propio & Preferencias \\
Buscar un mejor trabajo & Preferencias \\
\hline
\end{tabular}

Fecha de recepción: Abril de 2008.

Fecha de aceptación: Septiembre de 2008. 